Documentation et bibliothèques

DOCUMENTATION BIBLIOTHËQUES

\title{
L'archivistique moderne : pour une efficacité administrative accrue
}

\section{Daniel Ducharme et Chaker Ghariani}

Volume 32, numéro 3, juillet-septembre 1986

URI : https://id.erudit.org/iderudit/1052675ar

DOI : https://doi.org/10.7202/1052675ar

Aller au sommaire du numéro

Éditeur(s)

Association pour l'avancement des sciences et des techniques de la

documentation (ASTED)

ISSN

0315-2340 (imprimé)

2291-8949 (numérique)

Découvrir la revue

Citer cet article

Ducharme, D. \& Ghariani, C. (1986). L'archivistique moderne : pour une efficacité administrative accrue. Documentation et bibliothèques, 32(3), 93-95.

https://doi.org/10.7202/1052675ar

Tous droits réservés (C) Association pour l'avancement des sciences et des techniques de la documentation (ASTED), 1986
Ce document est protégé par la loi sur le droit d'auteur. L'utilisation des services d'Érudit (y compris la reproduction) est assujettie à sa politique d'utilisation que vous pouvez consulter en ligne.

https://apropos.erudit.org/fr/usagers/politique-dutilisation/ 


\section{L'archivistique moderne: pour une efficacité administrative accrue}

II n'est un secret pour personne que l'on assiste présentement à l'émergence d'une nouvelle forme de société dans laquelle la majeure partie de la population active participe directement ou indirectement à la création et à la diffusion d'informations de toutes formes. Cette société d'information est en quelque sorte le résultat d'un mouvement qui a débuté avec l'ère industrielle et qui n'a fait que s'accélérer depuis, amenant comme conséquence première la croissance phénoménale de l'administration. Celle-ci, à son tour, a pour corrolaire inévitable l'explosion de l'information. Explosion quantitative, d'une part, en raison de la masse documentaire produite par l'administration et qualitative, d'autre part, en ce qu'elle oblige à repenser son mode de conservation et d'exploitation ${ }^{1}$. Ce qu'on appelle "virage technologique» (informatique, bureautique, télématique, etc) n'est rien d'autre que l'expression de ce phénomène.

En dépit des moyens mis à sa portée, l'entreprise n'est pas toujours en mesure d'éviter la dégradation des services administratifs chargés de harnacher l'explosion de l'information. En effet, l'entreprise est littéralement débordée par l'énorme quantité de documents qu'elle doit ellemême produire. Ces documents et, partant, cette information, constituent sa mémoire. Pour donner une continuité à son action et pour assurer des fondements motivés à ses décisions, elle doit constamment y recourir ${ }^{2}$. Il y va du maintien de son efficacité administrative.

Il est donc impératif de réactiver cette fonction mémoire et d'organiser rationnellement le chaos causé par l'abondance de l'information. En effet, trop de documents utiles se perdent faute de moyens de repérage et trop de documents inutiles sont conservés, gênant ainsi l'activité des administrateurs. C'est précisément ici qu'intervient l'archivistique moderne.

Dans le présent article ${ }^{3}$, nous nous proposons de démontrer la pertinence de l'intervention archivistique face aux problèmes ci-devant mentionnés. Pour ce faire, nous examinerons trois composantes de cette intervention et nous souli- gnerons en quoi elles amènent une plus grande efficacité administrative de l'entreprise.

\section{Cadre théorique et précisions terminologiques}

Les archives se définissent par l'ensemble des documents qui résultent de l'activité d'une entreprise. On peut considérer cette définition comme axiomatique. En est-il ainsi de la notion d' 'sarchivistique moderne»? II s'agirait essentiellement d'une pratique appliquée non seulement aux documents inactifs, comme on le croyait naguère, mais également à l'ensemble des documents dès leur production et durant toute leur existence. Une telle vision constitue une approche globale: elle «intègre l'ensemble des opérations liées à l'administration des documents dans un but d'exploitation»4; elle unit deux volets (gestion des documents et archives historiques) d'une seule et même discipline. À la lumière de cette approche, l'archivistique comprendrait donc l'étude de toutes les activités liées à la création, à la sélection, au traitement et à l'exploitation des documents quels que soient leur âge et leur nature.

L'efficacité administrative se définit, quant à elle, par tout ce qui se répercute positivement sur les prestations d'une administration. II s'agit donc d'une notion beaucoup plus large que celle de rentabilité qui n'en est qu'une composante. Rien d'étonnant alors qu'elle traduise aussi des préoccupations telles la gestion rationnelle de l'espace, la communication de l'information et la prise de décision.

\section{L'archivistique: outil de contrôle de la planification}

Avant d'expliquer en quoi l'intervention archivistique se traduit par une plus grande efficacité administrative pour l'entreprise, nous fixerons sa sphère d'influence. En fait, elle s'inscrit au coeur du processus de planification des activités de l'entreprise. La planification suppose un certain nombre d'éléments de base: définition des objec-
1. Pour le développement de cette idée, voir Bruno Delmas, "L'administration et ses archives", International Journal of Archives, vol. 1, no. 2 (1980), 14.

2. Ibid, 15

3. Ce texte a été réalisé sous la direction de Carol Couture, professeur agrégé à l'École de bibliothéconomie et des sciences de l'information, dans le cadre d'un séminaire de recherche en archivistique (BLT-6423).
4. Jacques Ducharme et Jean-Yves Rousseau, "L'interdépendance des archives et de la gestion des documents: une approche globale de l'archivistique», Archives, vol. 12, no 1 (1980), 21 
tifs, mise en oeuvre des politiques pour les atteindre, prévision de l'imprévisible et réduction maximum de l'arbitraire. Aussi l'entreprise doitelle se doter d'un système de contrôle adéquat, c'est-à-dire d'un système d'information qui permette l'identification des faiblesses de fonctionnement et, en conséquence, les solutions à proposer. Voilà pourquoi un système d'information s'avère un outil indispensable de régulation et de contrôle de la planification. C'est précisément à ce niveau que l'archivistique a un rôle prépondérant à jouer ${ }^{5}$.

Pour illustrer l'idée développée ci-haut, prenons l'exemple d'une administration qui, pour une raison ou pour une autre, est contrainte de modifier les objectifs d'un programme. Deux options s'imposent: entreprendre de nouvelles études afin d'identifier les corrections nécessaires, ce qui suppose des dépenses supplémentaires; ou encore puiser dans les archives des études de programmes antérieurs similaires. L'archiviste, par le biais du système d'information, peut de la sorte aider l'administration à faire le choix approprié.

\section{La théorie des trois âges}

L'intervention archivistique, pour aller dans le sens de l'efficacité administrative, doit reposer, à notre avis, sur la théorie des trois âges, laquelle s'inscrit parfaitement dans la logique développée ci-dessus. Cette théorie permet de mettre l'accent sur la continuité du document; elle permet d'en suivre le cheminement depuis sa création jusqu'à son élimination. Son incorporation aux archives historiques ne marque pas sa fin mais bien une autre étape dans le processus de traitement.

La théorie des trois âges peut se résumer comme suit: la vie d'un document est divisée en une phase active, une phase semi-active et une phase inactive. Il est à un stade actif s'il est indispensable au fonctionnement courant de l'administration. Son utilisation s'avère fréquente et, en conséquence, il doit demeurer physiquement près des usagers. II est semi-actif s'il ne sert qu'occasionnellement. Son utilisation étant moins fréquente, il pourra être entreposé ailleurs que dans les espaces administratifs. Enfin, le docu- ment est inactifs'il n'a plus d'utilité prévisible pour l'administration.

La théorie des trois stades, clef de voûte de l'archivistique moderne, sous-tend les trois composantes de toute politique de traitement des documents $^{6}: 1$ ) la production des documents, 2) le système de classification, et 3) le calendrier de conservation.

\section{La production des documents}

Pour les profanes, il pourra sembler étrange que l'intervention archivistique se manifeste dès la création du document. N'avions-nous pas coutume de considérer l'archivistique comme un intervenant qui ne s'occupe que des documents existants? L'administration a toutefois avantage à considérer son initiative dès la création: elle permettra de prévoir la conception, la diffusion, la réception des documents et d'éviter ainsi bon nombre d'erreurs coûteuses par la suite. En effet, une intervention dès la conception concourt à enrayer le flot des documents inutiles puisqu'elle permet de mesurer les besoins réels des services administratifs. De plus, elle facilitera le gommage de renseignements inutiles sur les documents et les ajouts utiles. L'intervention de l'archiviste s'inscrit dans un effort de normalisation de la présentation des documents: à cet effet, on devra choisir une qualité de papier en rapport avec l'importance du document?

De tout cela, il résultera une économie réelle et une augmentation de l'efficacité administrative pour l'entreprise. Mais, avant tout, la rationalisation de la création des documents s'inscrit dans l'effort de planification de l'entreprise et elle se répercutera - nous le verrons plus loin - sur l'intervention archivistique dans son ensemble.

\section{Le système de classification}

Le guide de classement se présente comme un outil de travail; il n'est rien d'autre que la concrétisation d'un système de classification dont les avantages, pour l'administration, sont multiples.
5. Ibid, 22-23.
6. Ces éléments sont de deux types: éléments constitutifs (la création, le traitement des documents actifs, semi-actifs et essentiels) et de soutien (inventaire, calendrier de conservation). Voir Carol Couture et Jean-Yves Rousseau, Les archives au XXe siècle, Montréal, Université de Montréal, Secrétariat général, service des archives, 1982, Première partie, chapitre 3 .

7. Ibid, p. 47-50, et T.R. Schellengerg, Modern archives principles and techniques. Melbourne, F.W. Cheshire, 1956, chapitre $1 \mathrm{~V}$. 
Toute administration, quelle qu'elle soit, classe ses documents. Cela lui permet de profiter au maximum de l'information consignée sur les supports de toute nature. Mais l'intervention archivistique, par le biais du guide de classement, accélère la mise en ordre des documents, et par le fait même, son repérage quand le besoin s'en fait sentir. De plus, dans la mesure où il facilite la localisation rapide non seulement de l'information en général mais surtout de l'information la plus pertinente, il accélère également la prise de décision éclairée. Cela représente un atout important pour l'efficacité administrative.

Le guide de classement, entre autres avantages, atténue les craintes de l'administration devant l'extrême mobilité du personnel de bureau puisqu'il assure une stabilité et un suivi dans le classement et le repérage de l'information. Ainsi, une secrétaire appelée à travailler, pour une raison ou pour une autre, dans un service voisin n'éprouvera pas de difficultés à s'adapter puisqu'elle sera déjà familière avec le guide. De tout cela, il résultera une diminution de l'arbitraire au cours du processus de prise de décision. L'adoption d'un guide de classement pas l'administration permet également l'utilisation rationnelle de l'espace et donc une diminution des coûts. Un tel instrument facilitera aussi le traitement de la masse documentaire.

\section{Le calendrier de conservation}

Le calendrier de conservation doit être considéré comme la pierre angulaire d'une archivistique efficace. Comme le guide de classement, il s'agit d'un instrument de travail. Fondé sur la théorie des trois âges, le calendrier détermine l'itinéraire d'un document tout au long de son cycle de vie. Grâce aux règles de conservation, il permet de fixer la période de conservation des documents et de «déterminer le degré d'importance accordé à l'information qu'ils contiennent»8. Ainsi, on ne conserve pas un simple communiqué aussi longtemps qu'un procès-verbal et, de la sorte, on a une indication du degré d'importance de chacun d'eux.

Le calendrier de conservation permet la diminution de la masse documentaire; il aide l'administration à traiter adéquatement chaque type de document au moment approprié. Ainsi, seuls les documents pertinents sont retenus. De plus, il favorise l'élimination des documents dont la fréquence de consultation ou la valeur ne justifient pas la conservation dans les bureaux; il en résulte un allégement des classeurs et leur utilisation pour des documents plus utiles et plus utilisés. II permet également un gain de temps, donc de personnel, dans la mesure où un calendrier bien appliqué permet de repérer le document recherché, qu'il soit actif, semi-actif ou inactif. Ainsi, le personnel retrouvera plus facilement un document courant puisque celui-ci se trouve isolé des dossiers semi-actifs ou inactifs. Enfin, le calendrier de conservation entraîne des économies d'espace et de matériel, en ce qu'il facilite l'élimination rapide des documents dont la période de conservation est échue. Par conséquent, on utilise au maximum le matériel existant (classeurs, chemises, etc.)

\section{Conclusion}

Si nous avons retenu, comme principaux éléments de l'archivisque moderne, les interventions au stade de la création des documents, le système de classification et le calendrier de conservation, c'est qu'ils permettent, autant que faire se peut, de prévoir l'imprévisible en réduisant au maximum l'arbitraire. De plus, ces éléments permettent d'exercer un contrôle permanent sur la pertinence de l'information et de la bien situer à l'intérieur de la théorie des trois âges. C'est surtout en ce sens que l'archivistique moderne est une véritable science de l'information, utile autant à l'administration qu'à la recherche.

Si l'archivistique ne peut, à elle seule, accoître le chiffre d'affaires de l'entreprise, elle permet, à tout le moins, d'en rentabiliser certaines activités et d'en réduire ainsi certains coûts d'exploitation au niveau des espaces, des équipements et du personnel.

Si l'administrateur n'est pas toujours sensible à l'argument de l'efficacité (décision, gestion, etc.), il l'est toujours à celui du profit.

\section{Daniel Ducharme \\ Chaker Ghariani}

École de bibliothéconomie et

des sciences de l'information

Université de Montréal

8. Abdelmajid Ferchichi et al., "Le calendrier de conservation: clé de voûte d'une archivistique efficace", in L'archivistique, une science de l'information en plein essor, Montréal, École de bibliothéconomie et des sciences de l'information, avril 1985. p. 4

9. Marcel Caya, «Rentabilité et gestion des documents», Archives vol. 14, no 4 (mars 1983), 13-14. 